\title{
Quercetin-induced autophagy flux enhances TRAIL-mediated tumor cell death
}

\author{
JI-HONG MOON $^{1,2}$, SEONG KUG EO ${ }^{1,2}$, JOHN HWA LEE ${ }^{1,2}$ and SANG-YOUEL PARK ${ }^{1,2}$ \\ ${ }^{1}$ Biosafety Research Institute, College of Veterinary Medicine, ${ }^{2}$ Department of Bioactive Material Sciences and \\ Research Center of Bioactive Materials, Chonbuk National University, Jeonju, Jeonbuk 561-756, Republic of Korea
}

Received April 13, 2015; Accepted May 4, 2015

DOI: 10.3892/or.2015.3991

\begin{abstract}
Quercetin is a potent cancer therapeutic agent and dietary antioxidant present in fruit and vegetables. Quercetin prevents tumor proliferation by inducing cell cycle arrest and is a well known cancer therapeutic agent and autophagy mediator. We investigated whether quercetin enhances TRAIL-induced tumor cell death and the possible mechanism in human lung cancer cells. We identified that quercetin markedly enhanced TRAIL-mediated lung cancer cell death. Quercetin treatment dose-dependently decreased the p62 protein expression and increased GFP-LC3B. Autophagy flux inhibitor, chloroquine treatment blocked the enhancing effects of TRAIL-induced apoptosis by quercetin. Our results indicated that quercetin enhanced TRAIL-induced cell death via autophagy flux activation, and also suggest that quercetin may be a therapeutic agent against human lung cancer via combination therapy with many anticancer drugs including TRAIL.
\end{abstract}

\section{Introduction}

Quercetin is a member of the flavonoids family and one of the leading dietary antioxidants. It is present ubiquitously in vegetables and fruits and is considered to exert beneficial health effects $(1,2)$. Quercetin is known to induce autophagy in several cell types (3-5) and has attracted much attention in recent years due to its anticancer effects in many types of cancer $(6,7)$.

Apoptosis is a morphologically obvious form of programmed cell death that plays a critical role during homeostasis, development, and diseases including cancer, acquired immunodeficiency syndrome and neurodegenerative disorders (8). Apoptosis, also called programmed cell death type I, can be induced in cancer cells by anticancer agents $(9,10)$.

Correspondence to: Professor Sang-Youel Park, Biosafety Research Institute, College of Veterinary Medicine, Chonbuk National University, 567 Baekje-Daero, Deokjin-gu, Jeonju, Jeonbuk 561-756, Republic of Korea

E-mail: sypark@chonbuk.ac.kr

Key words: quercetin, TRAIL, autophagy, lung cancer
Tumor necrosis factor (TNF)-related apoptosis-inducing ligand (TRAIL) was first confirmed as one of the TNF superfamily. It can induce apoptosis selectively in tumorigenic or transformed cells, but not in normal cells, highlighting its potential therapeutic application in cancer treatment (11).

Autophagy is a conserved trafficking pathway that is highly regulated by environmental conditions (12). The implication of autophagy as a cell death mechanism in cancer with inactivated apoptosis is not surprising. However, the role of autophagy in cancer and treatment responsiveness is complicated (13). Autophagy is a general term for the degradation of cytoplasmic ingredients within lysosomes (14). Macroautophagy was first described in mammals as the isolation of complete portions of cytosol, including soluble proteins as well as complete organelles, in a double membrane vesicle called autophagosome $(15,16)$. Once the 2 sides of the autophagosome fuse to each other, there is a second fusion event between the autophagosome and the lysosomes/vacuole that leads to the formation of the autolysosome (17). The anti-malarial drug, chloroquine (CQ) inhibits lysosomal acidification and prevents the degradation of autophagosomes, thereby suppressing the autophagy flux (18).

Microtubule-associated protein light chain 3 (LC3) is localized and aggregated on the autophagosome and is, therefore, considered as a marker of autophagy. LC3B undergoes lipidation and is recruited to the phagophore where it is essential for membrane elongation and closure (19). LC3B transforms from LC3B-I to LC3B-II during autophagosome formation (20). P62 is a multifunctional signaling molecule, associated with a variety of cellular pathways. It is one of the best-known autophagic substrates, and is, therefore, extensively employed as an indicator of autophagic degradation (21). P62 can deliver ubiquitinylated cargos to the proteasome, though they are mainly degraded by autophagy $(21,22)$. P62 levels are generally inversely related to autophagic degradation, since the loss of Atg genes or factors required for the fusion of autophagosomes with lysosomes all result in a marked increase of P62-positive aggregates $(23,24)$.

Apoptosis is blocked in various cancer cells, and autophagy may be a major contributing mechanism of cancer cell death; thus, the induction of autophagy may be employed as a promising therapeutic strategy in cancer treatment (25). A549, human lung cancer cells, are known to be TRAILresistant cells (26). We identified that treatment with TRAIL 
or quercetin alone did not exert an influence on A549 human cancer cells. We next investigated the effect of co-treatment of TRAIL and quercetin on human cancer cells and quercetinmediated autophagy flux. We also examined the effect of quercetin-induced autophagy on TRAIL-induced apoptosis in human lung cancer cells.

\section{Materials and methods}

Cell culture. The human lung cancer cell line A549 was obtained from the American Type Culture Collection (ATCC; Manassas, VA, USA). Cells were cultured in RPMI-1640 (Invitrogen-Gibco, Carlsbad, CA, USA) supplemented with $10 \%$ fetal bovine serum (FBS; Invitrogen-Gibco), $100 \mathrm{U} / \mathrm{ml}$ penicillin, and $0.1 \mathrm{mg} / \mathrm{ml}$ gentamycin in a humidified incubator maintained at $37^{\circ} \mathrm{C}$ and $5 \% \mathrm{CO}_{2}$. Cells were treated for $12 \mathrm{~h}$ with quercetin (Sigma-Aldrich, St. Louis, MO, USA) and then exposed for $3 \mathrm{~h}$ to $200 \mathrm{ng} / \mathrm{ml}$ TRAIL, with or without the autophagy inhibitor, chloroquine (10 $\mu \mathrm{M})$ (Sigma-Aldrich).

Crystal violet assay. Cell morphology was assessed microscopically (inverted Microscope, Nikon Eclipse TS100; Nikon Corp., Tokyo, Japan) and cell viability was determined by crystal violet staining (C0775; Sigma-Aldrich), as previously described (27). Briefly, cells were stained for $10 \mathrm{~min}$ at RT with crystal violet solution ( $0.5 \%$ crystal violet in $30 \%$ ethanol and 3\% formaldehyde), washed 5 times with water, and then dried. Subsequently, the cells were lysed with $1 \%$ SDS (sodium dodecyl sulphate) and the absorbance was measured at $550 \mathrm{~nm}$. Cell viability was calculated from the relative dye intensity of the samples compared to the controls.

Terminal deoxynucleotidyl transferase dUTP nick end labeling (TUNEL) assay. TUNEL assay was carried out as previously described (28). TUNEL analysis was performed to measure the degree of cellular apoptosis using an in situ ApoBrdU DNA fragmentation assay kit (BioVision, Mountain View, CA, USA), following the manufacturer's instructions. Cells were counterstained with propidium iodide (PI) to show cell nuclei.

Trypan blue exclusion assay. The number of viable cells was determined by trypan blue dye exclusion (Sigma-Aldrich) using a hemocytometer. The result was expressed as a percentage relative to vehicle-treated controls.

BacMam transduction. Wild-type or mutant GFP-tagged LC3B was expressed in cells by adding the appropriate concentrations of appropriate virus from the Premo Autophagy Sensor LC3B-GFP (BacMam 2.0) kit (P36235; Life Technologies) to the growth medium as indicated in the figure legends.

Western blot analysis. A549 cells were lysed in lysis buffer [25 mM HEPES (4-(2-hydroxyethyl)-1-piperazineethanesulfonic acid), $\mathrm{pH}$ 7.4, $100 \mathrm{mM} \mathrm{NaCl}, 1 \mathrm{mM}$ EDTA (ethylene diamine tetra acetic acid), $5 \mathrm{mM} \mathrm{MgCl}{ }_{2}, 0.1 \mathrm{mM}$ DTT (dithiothreitol), and a protease inhibitor mixture]. Whole cell proteins were electrophoretically resolved on a $10-15 \%$ sodium dodecyl sulfate polyacrylamide gel and transferred to a nitrocellulose membrane. Immunoreactivity was detected through sequential incubation with primary antibodies, horseradish peroxidase-conjugated secondary antibodies, and enhanced chemiluminescence reagents i.e. West Save Gold detection kit (AbFrontier Co., Ltd., Seoul, Korea). The primary antibodies used for immunoblotting were anti-LC3B (\#4108; Cell Signaling Technology, Danvers, MA, USA), anti-P62 (\#MABC32; Millipore, Billerica, MA, USA), antiphospho-AKT (\#2118-1; Epitomics, Burlingame, CA, USA), anti-caspase-3 (\#9665; Cell Signaling Technology), anticleaved caspase-3 (\#9661; Cell Signaling Technology) and anti- $\beta$-actin (A5441; Sigma Aldrich). Images were examined using a Fusion FX7 imaging system (Vilber Lourmat, Marne La Vallee, France). Densitometry of the signal bands was analyzed using Bio-1D software (Vilber Lourmat).

Statistical analysis. The unpaired t-test or Welch's correction was used for comparison between the 2 groups. The one-way ANOVA followed by the Tukey-Kramer test was used for multiple comparison. All statistical analyses were performed with GraphPad Prism software. Results were considered significant for values ${ }^{* *} \mathrm{P}<0.01$ or ${ }^{* * *} \mathrm{P}<0.001$.

\section{Results}

Quercetin enhanced TRAIL-mediated cell death in human lung cancer cells. We conducted several types of cell viability assays to investigate the effect of co-treatment with quercetin and TRAIL on A549 human lung cancer cells. First, we examined the photographed image of cell amounts using a light microscope and the crystal violet assay. The cell viability of cells treated with quercetin only was comparable to that of untreated controls. Cell death resulted in 3-5\% of TRAILtreated A549 cells. Importantly, quercetin treatment enhanced cell death to $\sim 70 \%$ on photographed images (Fig. 1A) and augmented cell death to $\sim 50 \%$ in the crystal violet assay (Fig. 1B and C). We additionally performed a TUNEL assay (Fig. 1D) and trypan blue exclusion assay (Fig. 1E). As shown in Fig. 1D, apoptosis in quercetin and TRAIL treated cells emitted green fluorescence indicative of DNA strand breakage. In Fig. 1E, quercetin dose-dependently increased TRAILmediated cell death. These results indicated that quercetin was effective in promoting TRAIL-induced cell death in A549 human lung cancer cells.

Quercetin treatment induces autophagy flux and apoptosis. We evaluated quercetin mediated autophagy flux by estimating LC3B transformation and P62 expression. Levels of the late autophagosome marker LC3-II increased in the quercetintreated group in a dose-dependent manner as compared with the control group on western blot analysis (Fig. 2A). The activation of the autophagy through the formation of autophagosomes in A549 lung cancer cells was visualized by the Premo Autophagy Sensor (LC3B-FP) BacMam 2.0 system. LC3B-FP and LC3B (G120A)-FP viral vectors (MOI=30) were transduced in A549 cells, enabling the expression of fluorescent LC3B protein. We consequently monitored autophagosomes dynamics using inverted fluorescent microscopy. The mutant chimera LC3B (G120A)-FP was used as a negative control. According to the results shown in Fig. 2B, BacMam LC3B (G120A)-FP transduced cells showed a marked diffuse cytosolic expres- 

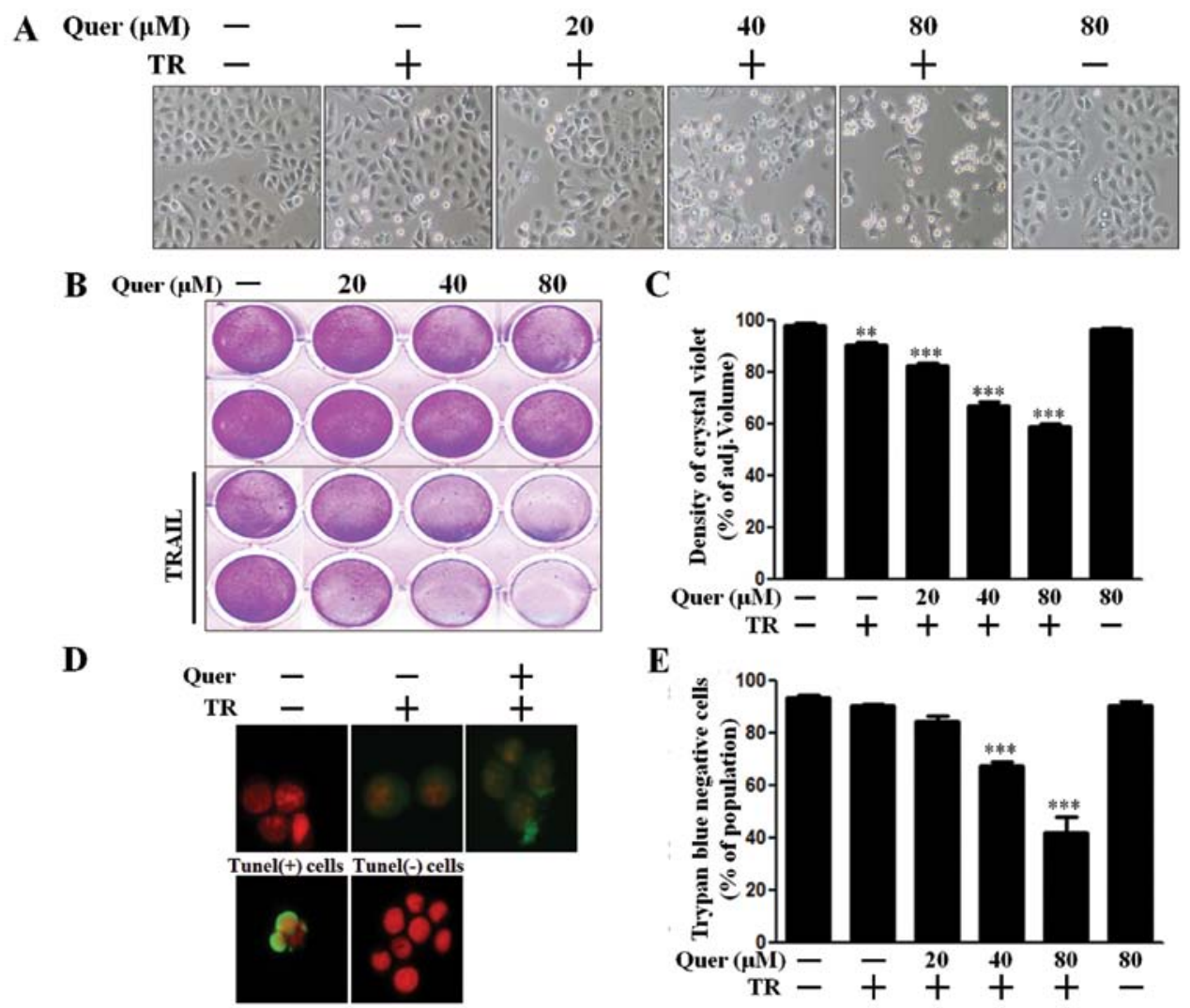

$\mathbf{E}$

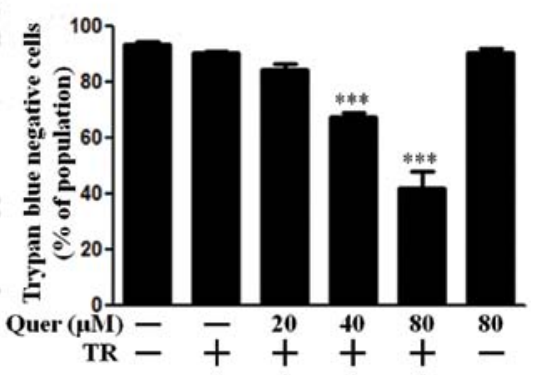

Figure 1. Quercetin enhanced TRAIL-mediated cell death in human lung cancer cells. A549 human lung cancer cells were pretreated with quercetin (12 h) in a dose-dependent manner, and then exposed to $200 \mathrm{ng} / \mathrm{ml}$ TRAIL for $3 \mathrm{~h}$. Cell viability was measured by photographed image with a light microscope (magnification, x100) (A) and by the crystal violet assay (B). (C) Bar graph indicated that the averages of density of dyed crystal violet. (D) Representative fluorescence images of TUNEL in A549 cells after co-treatment with $80 \mu \mathrm{M}$ of quercetin (12 h) and $200 \mathrm{mg} / \mathrm{ml}$ of TRAIL (3 h). The positive (green) cells were counterstained with PI (red) to show all cell nuclei. (E) The treated cells were also measured by the trypan blue dye exclusion assay. ${ }^{* *} \mathrm{P}<0.01,{ }^{* * * *} \mathrm{P}<0.001$; significant differences between control and each treatment group. Quer, quercetin; TR, tumor necrosis factor (TNF)-related apoptosis-inducing ligand.

A

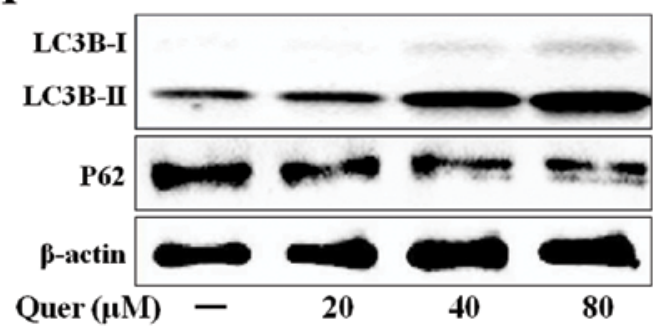

B Quer $(\mu \mathrm{M})-$

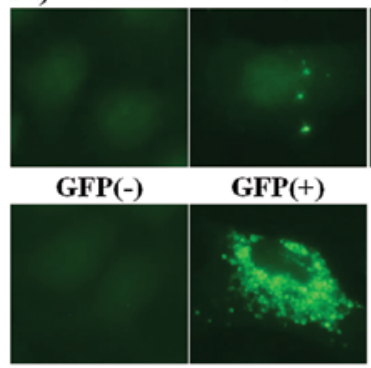

C
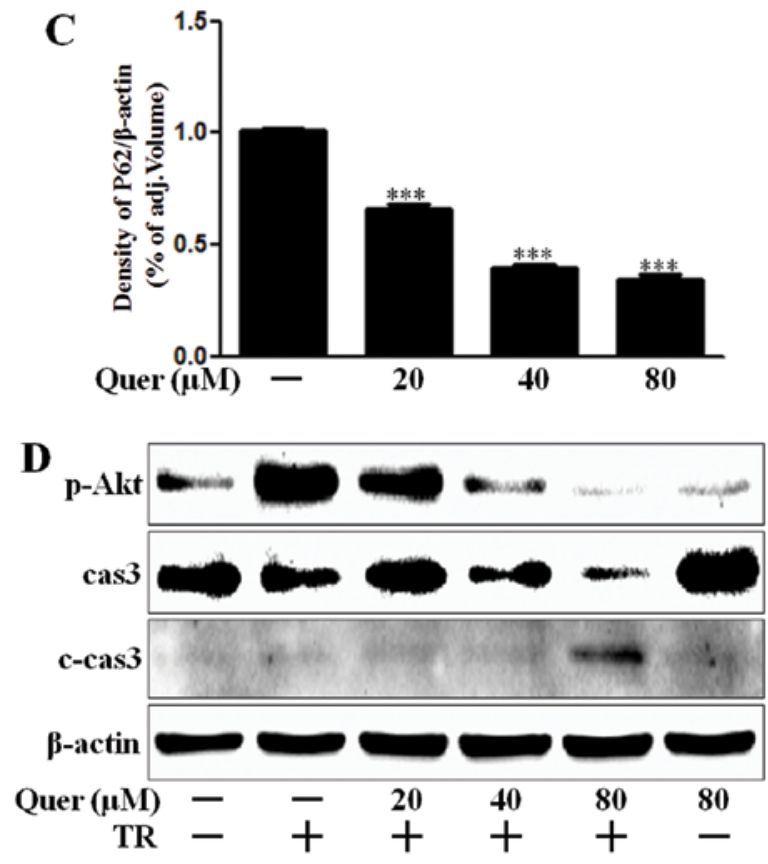

Figure 2. Quercetin treatment induces autophagy flux and apoptosis. (A) A549 cells were treated with quercetin (12 h) in a dose-dependent manner. The treated cells were assessed for LC3B production and P62 expression by western blot analysis. (B) A549 cells were mixed with a titration (30 MOI) of BacMam GFP-LC3B virus for $18 \mathrm{~h}$, and then treated with quercetin (12 h), negative control reagent and positive control reagent (CQ) at the same time. (C) Bar graph indicated the averages of P62/ $\beta$-actin ratio. (D) Cells were pre-treated with quercetin (12 h) in a dose-dependent manner, and then exposed to $200 \mathrm{ng} / \mathrm{ml}$ TRAIL for $1 \mathrm{~h}$. The treated cells were assessed for phosphorylation of Akt and caspase-3 cleavage by western blot analysis. Results were normalized with $\beta$-actin. ${ }^{* * * *} \mathrm{P}<0.001$; significant differences between control and each treatment group. Quer, quercetin; TR, tumor necrosis factor (TNF)-related apoptosis-inducing ligand; p-Akt, phosphorylation of Akt; cas3, caspase-3; c-cas3, cleaved caspase-3; adj.volume, adjustment of volume (band volume minus background volume). 
$\mathbf{A}$

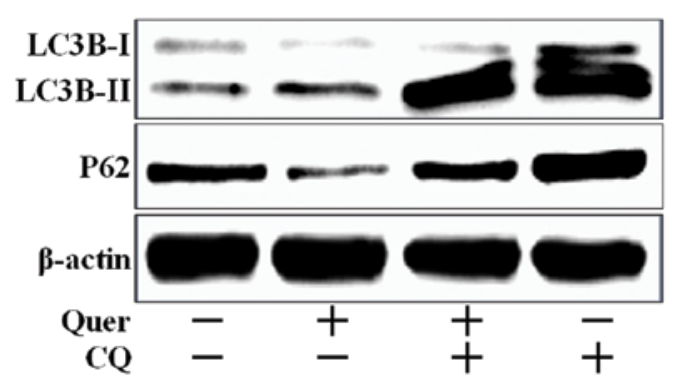

B

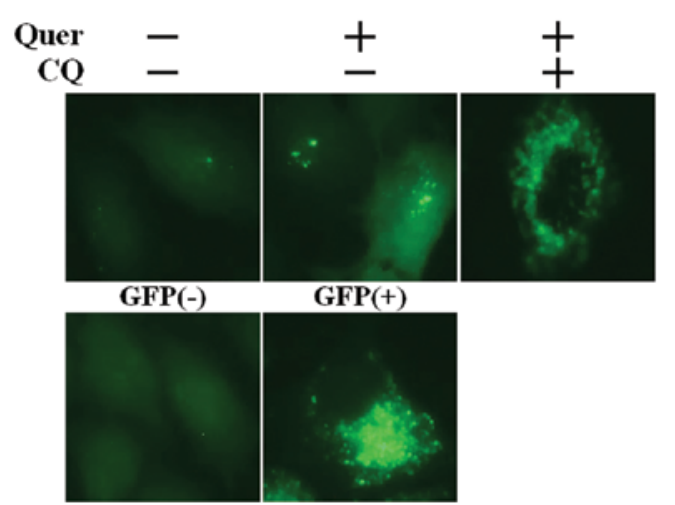

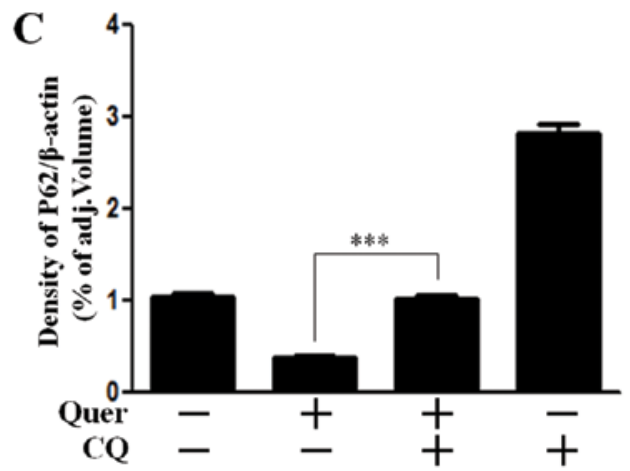

D

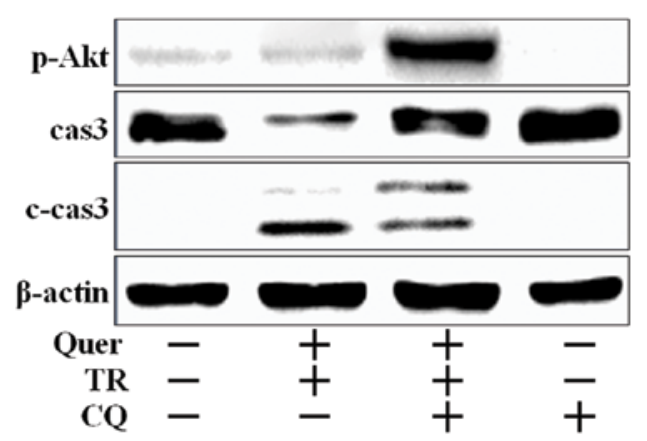

Figure 3. Quercetin regulates autophagy flux. (A) A549 cells were pretreated with autophagy inhibitor (chloroquine) (1 h) and then exposed to quercetin (12 h) in dose-dependent manner. The treated cells were assessed for LC3B production and P62 expression by western blot analysis. (B) A549 cells were mixed with a titration (30 MOI) of BacMam GFP-LC3B virus over $18 \mathrm{~h}$, and then treated with chloroquine for $1 \mathrm{~h}$ and quercetin for $12 \mathrm{~h}$. Negative control reagent and positive control reagent (CQ) was also treated at the same time. (C) Bar graph indicated the averages of P62/ $\beta$-actin ratio. (D) Cells were pre-treated with autophagy inhibitor (chloroquine) $(1 \mathrm{~h}$ ) and then exposed to quercetin for $12 \mathrm{~h}$ and TRAIL for $1 \mathrm{~h}$. The treated cells were assessed for phosphorylation of Akt and caspase- 3 cleavage by western blot analysis. Results were normalized with $\beta$-actin. ${ }^{* * *} \mathrm{P}<0.001$; significant differences between control and each treatment group. Quer, quercetin; TR, tumor necrosis factor (TNF)-related apoptosis-inducing ligand; CQ, chloroquine; p-Akt, phosphorylation of Akt; cas3, caspase-3; c-cas3, cleaved caspase-3; adj.volume, adjustment of volume (band volume minus background volume).

sion pattern. A549 cells treated with quercetin, exhibited an extensive punctate fluorescent distribution pattern, suggesting LC3B-FP protein accumulation in autophagosome. P62 was also decreased dose-dependently by quercetin (Fig. 2A and C). These results suggested that quercetin dose-dependently mediated autophagy flux. Akt is a key signaling molecule that associates oncogenic receptors to many essential pro-survival cellular functions in human cancer (29). We determined that quercetin treatment interrupted Akt activation and enhanced caspase-3 cleavage (Fig. 2D). Caspase-3 plays a key role in regulating programmed cell death or apoptosis, a normal process required for regulatory maintenance of physiological functions (30). Thus, these results suggested that quercetin mediated autophagy flux and enhanced TRAIL-induced apoptosis in A549 human lung cancer cells.

Quercetin regulates autophagy flux. We investigated whether quercetin induced autophagy flux or regulated chloroquine (CQ)-mediated autophagy flux. CQ is widely used to inhibit the maturation of autophagosome into degradative autolysosome $(31,32)$. We showed that upregulation of LC3B-II by quercetin was potentiated by $C Q$, since $C Q$ inhibited the fusion of autophagosome and autolysosome (Fig. 3A). A549 cells treated with quercetin, presented an extensive punctate fluorescent distribution pattern which was also augmented by CQ (Fig. 3B). P62 is a crucial mediator to target protein to the autophagy system in the removal of aggregated proteins. P62 is itself degraded during autophagy (33). Reduction of P62 level by quercetin treatment indicated autophagy flux activation, and also the activation of autophagy flux was inhibited by CQ treatment, given the increase of P62 levels (Fig. 3A and C). According to the results shown in Fig. 3D, A549 cells co-treated with CQ were augmented Akt activation and decreased caspase- 3 cleavage, whereas caspase- 3 activation were induced in cells treated with quercetin and TRAIL treatment, which indicated that CQ played a protective role against apoptotic cell death in A549 cells.

Autophagy regulates quercetin-induced TRAIL sensitivity. We analyzed cell viability using CQ, to investigate the effect of quercetin-mediated autophagy on TRAIL-sensitivity. A549 cells treated with CQ recovered Akt activation and were protected against cleavage of caspase-3 (Fig. 3B). We accordingly investigated whether autophagy inhibition using CQ exerted an influence on cell viability. We examined the photographed image of cell amounts using light microscopy and performed the crystal violet assay. Quercetin treatment in TRAIL treated cells enhanced cell death to $\sim 70 \%$ in the photographed image (Fig. 4A), and to $50 \%$ in the crystal violet assay (Fig. 4B and C). However, cell death recovered to $\sim 60 \%$ cell survival in the photographed image (Fig. 4A) and $85 \%$ cell survival in the crystal violet assay (Fig. 4B and C). We 
A
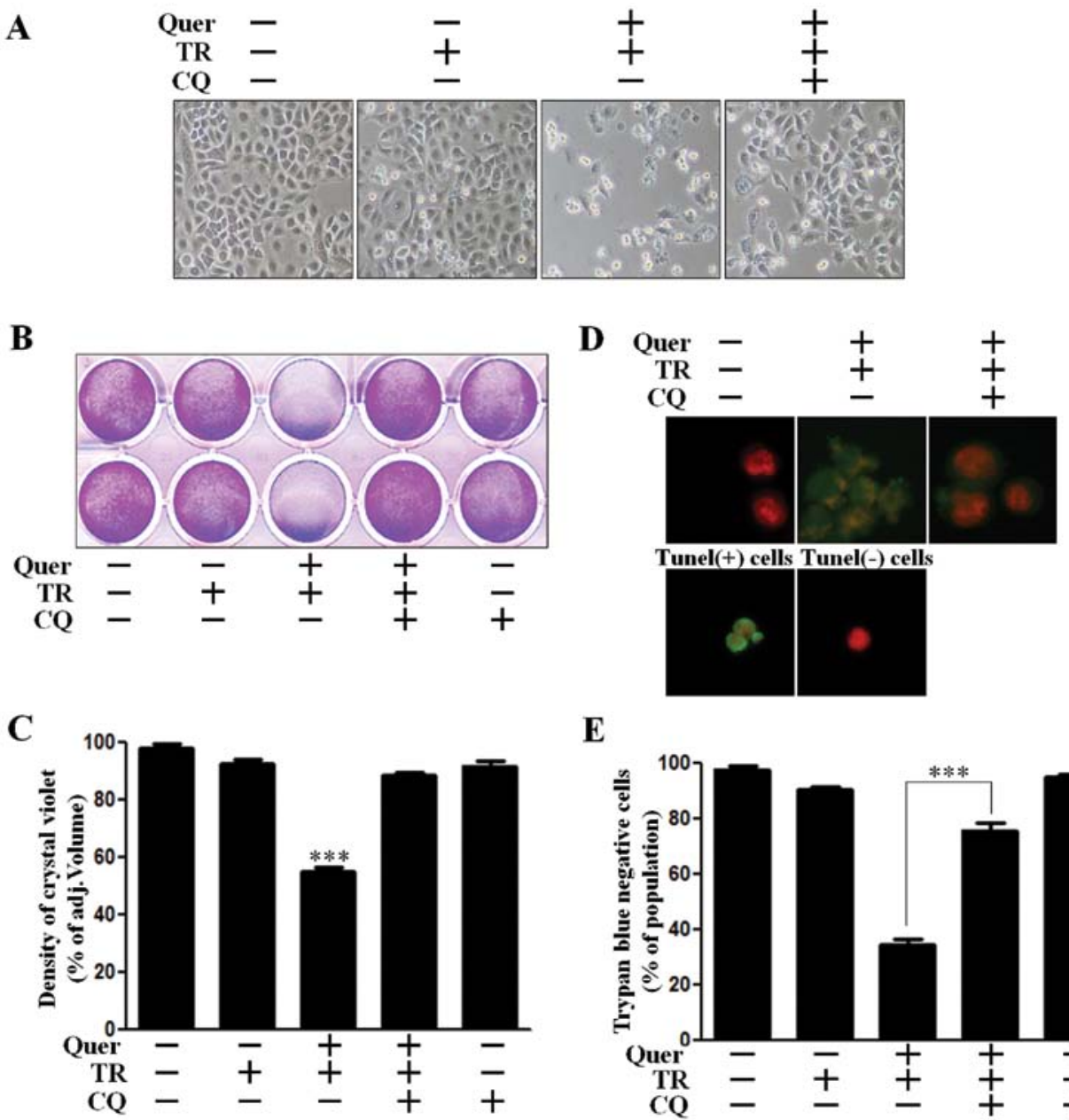

$\mathbf{E}$

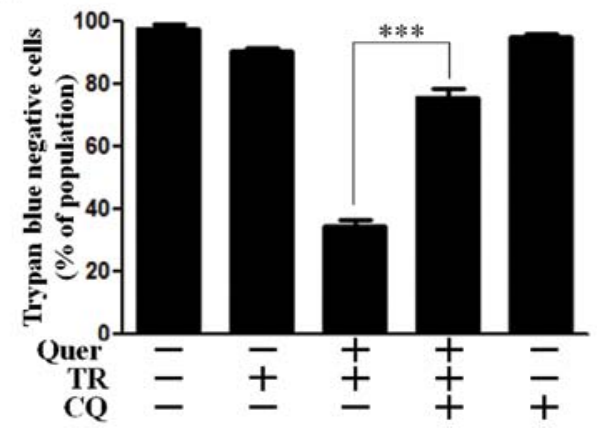

Figure 4. Autophagy regulates quercetin-induced TRAIL sensitivity. A549 human lung cancer cells were pretreated with CQ (1 h) and then exposed to quercetin $(12 \mathrm{~h}$ ) and TRAIL $(3 \mathrm{~h})$. Cell viability was measured by photographed image with a light microscope (magnification, x100) (A) and by the crystal violet assay (B). (C) Bar graph indicated the average density of dyed crystal violet. (D) Representative fluorescence images of TUNEL were implemented in A549 cells after the co-treatment $50 \mu \mathrm{M}$ of CQ $(1 \mathrm{~h}), 80 \mu \mathrm{M}$ of quercetin $(12 \mathrm{~h})$ and $200 \mathrm{mg} / \mathrm{ml}$ of TRAIL $(3 \mathrm{~h})$. The positive (green) cells were counterstained with $\mathrm{PI}$ (red) to show all cell nuclei. (E) The treated cells were also measured by trypan blue dye exclusion assay. ${ }^{* * * *} \mathrm{P}<0.001$; significant differences between control and each treatment group. Quer, quercetin; TR, tumor necrosis factor (TNF)-related apoptosis-inducing ligand; CQ, chloroquine.

performed a TUNEL assay (Fig. 1D) and trypan blue exclusion assay (Fig. 1E). As shown in Fig. 4D, the apoptotic process in quercetin and TRAIL treated cells emitted green fluorescence, indicative of DNA strand breakage, however, green fluorescence was weak in CQ-treated cells. In Fig. 1E, CQ treatment alleviated cell death by inhibiting quercetin-mediated TRAIL sensitivity. These results indicated that CQ was effective in inhibiting quercetin-induced TRAIL sensitivity by regulating autophagy flux in A549 human lung cancer cells. Collectively our data suggested that quercetin-induced autophagy flux had a harmful effect on TRAIL sensitivity and inhibition of autophagy flux played a protective role against quercetinmediated TRAIL sensitivity in A549 human lung cancer cells.

\section{Discussion}

The purpose of the present study was to investigate the role of quercetin-induced autophagy flux and the regulation of TRAIL-mediated sensitivity by quercetin treatment in A549 human lung cancer cells. The results suggested that quercetin-induced autophagy and the resultant enhancement in quercetin-induced TRAIL sensitivity might be a key underlying mechanism of autophagy flux.

Quercetin belongs to an extensive class of polyphenolic flavonoid compounds and is practically ubiquitous in plants and plant food sources. It has been studied as a promising chemoprevention component in a variety of cancer models (34). Consistent with previous reports, the present study showed that quercetin promoted TRAIL-mediated apoptosis in lung cancer cells.

Autophagy is an evolutionary conserved, dynamic lysosome-mediated process that entails the sequestration and delivery of cytoplasmic material to the lysosome where it is degraded and recycled $(35,36)$. Some studies have insisted that autophagy is a double-edged sword, with both useful and harmful potential in cancer (37). Wang et al (38) suggested that quercetin induces protective autophagy in gastric cancer cells. Their study indicated that quercetin-induced apoptosis and autophagy played a protective role during apoptosis. The present study, on the other hand, showed that quercetininduced autophagy induced TRAIL-sensitivity, and then mediated apoptosis. We demonstrated that quercetin-induced 
autophagy did not play a protective function by using an autophagy inhibitor in A549 lung cancer cells. However, this experimental evidence was insufficient to elucidate function of autophagy flux.

Some reports confirmed that TRAIL induces autophagy in several types of cancer cells $(39,40)$. However, our results indicated that TRAIL treatment did not mediate transformation from LC3B-I to LC3B-II (data not shown). On the contrary, TRAIL-treated LC3-II transformation decreased slightly than control. We concluded that TRAIL was possibly not associated with autophagy in A549 lung cancer cells.

According to the results reported in Figs. 2D and 3D, Akt signaling was activated by TRAIL treatment and decreased activation of Akt was observed on treatment with quercetin. Akt signal is activated by TRAIL-resistance in breast cancer cells (41) and TRAIL phosphorylated PI3k and Akt in leukemic T Jurkat cells (42). Akt or protein kinase B (PKB) is one of the most critical kinases in the regulation of cell survival. Enhanced activity of the PI3K/Akt pathway is found in many malignancies and is associated with the stimulation of cell growth and cell survival (43).

Cross-talk between autophagy and apoptosis is complicated and sometimes contradictory; however, it is a critical determinant of the overall fate of the cell. This study determined that quercetin-induced autophagy flux might play a crucial role in TRAIL sensitivity in A549 human lung cancer cells. Quercetin may thus be a useful regulator for TRAILmediated cancer therapy in lung cancer.

\section{Acknowledgements}

The present study was supported by a grant from the National Research Foundation of Korea (NRF), funded by the Korean government (MISP) (2013R1A4A1069486).

\section{References}

1. Baowen Q, Yulin Z, Xin W, Wenjing X, Hao Z, Zhizhi C, Xingmei D, Xia Z, Yuquan W and Lijuan C: A further investigation concerning correlation between anti-fibrotic effect of liposomal quercetin and inflammatory cytokines in pulmonary fibrosis. Eur J Pharmacol 642: 134-139, 2010.

2. Boots AW, Haenen GR and Bast A: Health effects of quercetin: From antioxidant to nutraceutical. Eur J Pharmacol 585: 325-337, 2008.

3. Qu L, Liang X, Gu B and Liu W: Quercetin alleviates high glucose-induced Schwann cell damage by autophagy. Neural Regen Res 9: 1195-1203, 2014.

4. Kim H, Moon JY, Ahn KS and Cho SK: Quercetin induces mitochondrial mediated apoptosis and protective autophagy in human glioblastoma U373MG cells. Oxid Med Cell Longev 2013: 596496, 2013.

5. Wei L, Liu JJ, Cao J, Du NC, Ji LN and Yang XL: Role of autophagy in quercetin-induced apoptosis in human bladder carcinoma BIU-87 cells. Zhonghua Zhong Liu Za Zhi 34: 414-418, 2012 (In Chinese).

6. Wei YQ, Zhao X, Kariya Y, Fukata H, Teshigawara K and Uchida A: Induction of apoptosis by quercetin: Involvement of heat shock protein. Cancer Res 54: 4952-4957, 1994.

7. Murakami A, Ashida $\mathrm{H}$ and Terao J: Multitargeted cancer prevention by quercetin. Cancer Lett 269: 315-325, 2008.

8. Steller H: Mechanisms and genes of cellular suicide. Science 267: 1445-1449, 1995.

9. Hickman JA: Apoptosis induced by anticancer drugs. Cancer Metastasis Rev 11: 121-139, 1992.

10. Zhang JY: Apoptosis-based anticancer drugs. Nat Rev Drug Discov 1: 101-102, 2002.
11. MacFarlane M: TRAIL-induced signalling and apoptosis. Toxicol Lett 139: 89-97, 2003.

12. Wang CW and Klionsky DJ: The molecular mechanism of autophagy. Mol Med 9: 65-76, 2003.

13. Chen N and Karantza V: Autophagy as a therapeutic target in cancer. Cancer Biol Ther 11: 157-168, 2011.

14. Cuervo AM: Autophagy: In sickness and in health. Trends Cell Biol 14: 70-77, 2004.

15. Seglen PO, Berg TO, Blankson H, Fengsrud M, Holen I and Strømhaug PE: Structural aspects of autophagy. Adv Exp Med Biol 389: 103-111, 1996.

16. Mortimore GE, Miotto G, Venerando R and Kadowaki M: Autophagy. Subcell Biochem 27: 93-135, 1996.

17. Cuervo AM: Autophagy: Many paths to the same end. Mol Cell Biochem 263: 55-72, 2004.

18. Liu B, Bao JK, Yang JM and Cheng Y: Targeting autophagic pathways for cancer drug discovery. Chin J Cancer 32: 113-120, 2013.

19. Klionsky DJ: Autophagy: From phenomenology to molecular understanding in less than a decade. Nat Rev Mol Cell Biol 8: 931-937, 2007.

20. Rubinsztein DC, Cuervo AM, Ravikumar B, Sarkar S, Korolchuk V, Kaushik S and Klionsky DJ: In search of an 'autophagomometer'. Autophagy 5: 585-589, 2009.

21. Sahani MH, Itakura E and Mizushima N: Expression of the autophagy substrate SQSTM1/p62 is restored during prolonged starvation depending on transcriptional upregulation and autophagy-derived amino acids. Autophagy 10: 431-441, 2014.

22. Myeku N and Figueiredo-Pereira ME: Dynamics of the degradation of ubiquitinated proteins by proteasomes and autophagy: Association with sequestosome 1/p62. J Biol Chem 286: 22426-22440, 2011.

23. Bartlett BJ, Isakson P, Lewerenz J, Sanchez H, Kotzebue RW, Cumming RC, Harris GL, Nezis IP, Schubert DR, Simonsen A, et al: p62, $\operatorname{Ref}(2) \mathrm{P}$ and ubiquitinated proteins are conserved markers of neuronal aging, aggregate formation and progressive autophagic defects. Autophagy 7: 572-583, 2011.

24. Klionsky DJ, Abdalla FC, Abeliovich H, Abraham RT, AcevedoArozena A, Adeli K, Agholme L, Agnello M, Agostinis P, Aguirre-Ghiso JA, et al: Guidelines for the use and interpretation of assays for monitoring autophagy. Autophagy 8: 445-544, 2012.

25. Liu B, Cheng Y, Liu Q, Bao JK and Yang JM: Autophagic pathways as new targets for cancer drug development. Acta Pharmacol Sin 31: 1154-1164, 2010.

26. Jiang L, Hao JL, Jin ML, Zhang YG and Wei P: Effect of embelin on TRAIL receptor $2 \mathrm{mAb}$-induced apoptosis of TRAIL-resistant A549 non-small cell lung cancer cells. Asian Pac J Cancer Prev 14: 6115-6120, 2013.

27. Chaudhari AA, Seol JW, Lee YJ, Seol DW and Park SY: Hypoxia protects articular chondrocytes from thapsigargin-induced apoptosis. Biochem Biophys Res Commun 381: 513-517, 2009.

28. Seo JS, Moon MH, Jeong JK, Seol JW, Lee YJ, Park BH and Park SY: SIRT1, a histone deacetylase, regulates prion proteininduced neuronal cell death. Neurobiol Aging 33: 1110-1120, 2012.

29. Agarwal E, Brattain MG and Chowdhury S: Cell survival and metastasis regulation by Akt signaling in colorectal cancer. Cell Signal 25: 1711-1719, 2013.

30. Degterev A, Boyce M and Yuan J: A decade of caspases. Oncogene 22: 8543-8567, 2003.

31. Kroemer $\mathrm{G}$ and Jäättelä M: Lysosomes and autophagy in cell death control. Nat Rev Cancer 5: 886-897, 2005.

32. Boya P, González-Polo RA, Casares N, Perfettini JL, Dessen P, Larochette N, Métivier D, Meley D, Souquere S, Yoshimori T, et al: Inhibition of macroautophagy triggers apoptosis. Mol Cell Biol 25: 1025-1040, 2005.

33. Matsumoto G, Wada K, Okuno M, Kurosawa M and Nukina N: Serine 403 phosphorylation of p62/SQSTM1 regulates selective autophagic clearance of ubiquitinated proteins. Mol Cell 44: 279-289, 2011.

34. Jeong JH, An JY, Kwon YT, Rhee JG and Lee YJ: Effects of low dose quercetin: Cancer cell-specific inhibition of cell cycle progression. J Cell Biochem 106: 73-82, 2009.

35. Pattingre S, Tassa A, Qu X, Garuti R, Liang XH, Mizushima N, Packer M, Schneider MD and Levine B: Bcl-2 antiapoptotic proteins inhibit Beclin 1-dependent autophagy. Cell 122: 927-939, 2005.

36. Yang $\mathrm{Z}$ and Klionsky DJ: An overview of the molecular mechanism of autophagy. Curr Top Microbiol Immunol 335: $1-32,2009$. 
37. White E and DiPaola RS: The double-edged sword of autophagy modulation in cancer. Clin Cancer Res 15: 5308-5316, 2009.

38. Wang K, Liu R, Li J, Mao J, Lei Y, Wu J, Zeng J, Zhang T, Wu H, Chen L, et al: Quercetin induces protective autophagy in gastric cancer cells: Involvement of Akt-mTOR- and hypoxia-induced factor 1 $\alpha$-mediated signaling. Autophagy 7: 966-978, 2011

39. Singh K, Sharma A, Mir MC, Drazba JA, Heston WD, MagiGalluzzi C, Hansel D, Rubin BP, Klein EA and Almasan A: Autophagic flux determines cell death and survival in response to Apo2L/TRAIL (dulanermin). Mol Cancer 13: 70, 2014.

40. Park KJ, Lee SH, Kim TI, Lee HW, Lee CH, Kim EH, Jang JY, Choi KS, Kwon MH and Kim YS: A human scFv antibody against TRAIL receptor 2 induces autophagic cell death in both TRAIL-sensitive and TRAIL-resistant cancer cells. Cancer Res 67: 7327-7334, 2007.
41. Shankar E, Sivaprasad U and Basu A: Protein kinase C epsilon confers resistance of MCF-7 cells to TRAIL by Akt-dependent activation of $\mathrm{Hdm} 2$ and downregulation of p53. Oncogene 27: 3957-3966, 2008.

42. Zauli G, Sancilio S, Cataldi A, Sabatini N, Bosco D and Di Pietro R: PI-3K/Akt and NF-kappaB/IkappaBalpha pathways are activated in Jurkat T cells in response to TRAIL treatment. J Cell Physiol 202: 900-911, 2005.

43. Fresno Vara JA, Casado E, de Castro J, Cejas P, Belda-Iniesta C and González-Barón M: PI3K/Akt signalling pathway and cancer. Cancer Treat Rev 30: 193-204, 2004. 